POS PROCEEDINGS

\title{
The GRANDproto35 experiment
}

\section{QuanBu Gou*}

Key Laboratory of Particle Astrophysics, Institute of High Energy Physics, Chinese Academy of Sciences, Beijing 100049, China

E-mail: gougbei ihep.ac.cn

Olivier Martineau-Huynh, Jacques David, David Martin

LPNHE, CNRS-IN2P3 and Universités Paris 6 \& 7, BP200, 4 place Jussieu, 75252 Paris, France

Didier Charrier

SUBATECH, IN2P3-CNRS, Université de Nantes, Ecole des Mines de Nantes, Nantes, France

Sandra Le Coz, Junhua Gu, Xiangping Wu, Jianli Zhang

National Astronomical Observatory, Chinese Academy of Sciences, Beijing 100012, China

Zhen Wang, Yi Zhang, HongBo Hu

Key Laboratory of Particle Astrophysics, Institute of High Energy Physics, Chinese Academy of Sciences, Beijing 100049, China

\section{Valentin Niess}

Clermont Université,CNRS/IN2P3, LPC, BP. 10448, 63000 Clermond-Ferrand, France

\section{Charles Timmermans, Sijbrand de Jong, René Habraken}

Radboud University Nijmegen, Heyendaalseweg 135, 6525 AJ Nijmegen, The Netherlands and Nikhef, Science Park 105, 1098 XG Amsterdam, The Netherlands

\section{Ke Fang}

Joint Space-Science Institute, University of Maryland, College Park, MD, 20742, USA

\section{Kumiko Kotera,}

Institut d'Astrophysique de Paris, Sorbonne Université, UPMC Univ. Paris 6 and CNRS, UMR 7095, 98 bis bd Arago, 75014 Paris, France

\section{Washington Carvalho Jr.}

Physics Institute, University of Sao Paulo, Brazil

\section{Krijn De Vries}

Vrije Universiteit Brussel, Dienst ELEM, 1050 Brussels, Belgium

\section{Mauricio Bustamante}

Center for Cosmology and AstroParticle Physics, The Ohio State University, Columbus, OH 43210, USA

\section{Matías Tueros}


Instituto de Fisica La Plata, CONICE CCT-La Plata, La Plata, Argentina

\section{Clementina Medina}

Instituto Argentino de Radioastronomia, CONICET, La Plata, Argentina

The very low flux of ultra-high-energy cosmic-rays (UHECRs) requires detectors with a large effective area and high duty cycle to obtain a statistically relevant sample. Radio detection of extensive air showers (EAS) presents attractive aspects for future giant detectors of high energy cosmic particles, with very low cost per detection unit, easiness of deployment over large areas, and a duty cycle close to $100 \%$. However, autonomous detection of EAS -a necessary step towards the realization of this type of ambitious detectors- remains a challenge.

GRANDproto35 aims at demonstrating that radio-detection of air showers can be performed with very good background rejection, high efficiency, and an almost $100 \%$ duty cycle. The 35 GRANDproto antennas will perform a full measurement of the detected wave polarization. This makes GRANDproto35 uniquely qualified for the investigation of polarization characteristics of the radio emission from EAS, which may contribute to discriminate them from background signals. In addition, an array of 24 scintillators will allow offline cross-checks of the nature of the selected radio-candidates. We detail here the principle, progress and prospects of GRANDproto35, which serves as a step towards the Giant Radio Array for Neutrino Detection (GRAND) project. GRAND will consist of an array of $\sim 10^{5}$ radio antennas deployed over $\sim 200,000 \mathrm{~km}^{2}$ in mountainous sites.

35th International Cosmic Ray Conference - ICRC2017

10-20 July, 2017

Bexco, Busan, Korea

${ }^{*}$ Speaker. 


\section{Motivation for the GRANDproto35 project}

Radio detection of extensive air showers (EAS) induced by high energy $\left(\mathrm{E} \gtrsim 3 \cdot 10^{16} \mathrm{eV}\right)$ cosmic rays has made significant progress in the last decade [1]. In particular, the performance of radio arrays is now comparable to that of other established techniques for the determination of the EAS energy [2, 3, 4]. Studies of the maximum of the air shower development using radio [4, 5, 6] also rival existing techniques. However, most radio arrays used for EAS detection are externally triggered by particle detectors deployed at the same location. The main reason is that, outside polar areas, a wide variety of background sources (thunderstorms, HV lines, planes, etc.) are present in the surroundings of the radio array and emit transient electromagnetic signals in the frequency range where the radio detector is sensitive (few tens of $\mathrm{MHz}$ to few hundreds of $\mathrm{MHz}$ ). The background rate strongly depends on the local environment (typically, from tens of Hz/antenna, in the most remote areas, up to $\mathrm{kHz}$ or more), but it is always larger by several orders of magnitude than the rate of detectable cosmic rays.

A fully efficient, self-triggered radio array will be able to discriminate between these ultradominant backgrounds and EAS events. This requirement can only be fulfilled when efficient discriminating variables are defined. To this data analysis challenge can be added a technical challenge: implementing this background rejection at the antenna level and/or at the data acquisition level without introducing a large dead time of the detector or reducing its efficiency. So far, no radio array has satisfyingly solved this double issue. The current situation thus represents a limitation for the development of the technique, in particular for future self-triggered giant radio arrays such as GRAND [7]. Driven by the expertise acquired on previous setups $([8,9])$ we propose GRANDproto35, an experiment that aims to achieve autonomous radio-detection of EAS with high efficiency and excellent background rejection.

\section{The GRANDproto35 setup}

The GRANDproto35 detector will be composed of 35 antennas and 24 scintillators. The detection units are being deployed on the site of the 21CMA radio-interferometer [10], following a rectangular pattern spanning $800 \mathrm{~m}$ along the East-West axis and $2400 \mathrm{~m}$ along the North-South axis. This setup, shown in Figure 1, is optimized for the detection of EAS coming from the North. The GRANDproto35 detector benefits from the 21CMA infrastructure; in particular, from a $220 \mathrm{~V}$ wired power supply available at the foot of each detection unit, together with a network of optical fibers linking each detection unit to the central DAQ room. Therefore a large data throughput is available for each individual detector without being limited by power constraints.

\subsection{Radio Detectors}

The antennas of the GRANDproto35 radio units are active bow-tie antennas that follow a design very similar to the antennas used by the Pierre Auger Observatory in the Auger Engineering Radio Array (AERA) [11]. The main difference with the AERA detection unit is the vertical arm that was developed for GRANDproto35 antennas in addition to the two horizontal ones. The combined information of all three arms allows a full determination of the polarization of the signal (see section 3). Each of the three arms is equipped with a custom ASIC low-noise amplifier placed 


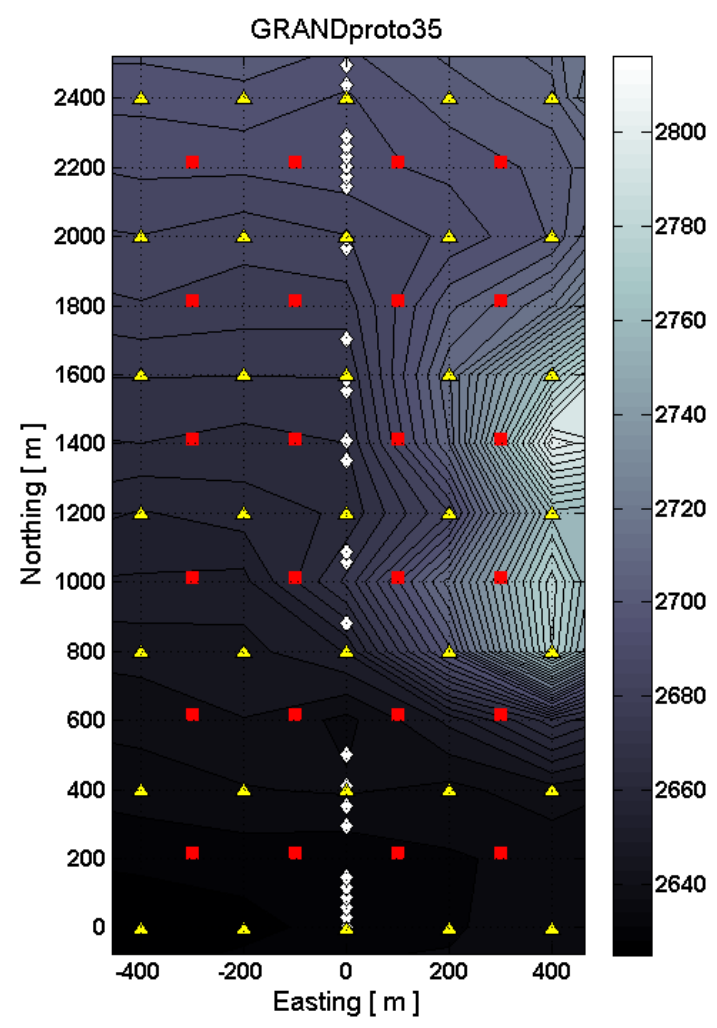

Figure 1: The GRANDproto35 layout, showing antennas (yellow triangles) and scintillators (red squares); Also showing the 21CMA pods (white diamonds). The color map shows the terrain elevation.

at the feed-point, from where the signal is sent to a front-end electronic (FE) board installed at the foot of the antenna. 32 of the 35 detection units of the GRANDproto35 radio array will be equipped with front-end boards developed at LPNHE Paris. On this board each of the three signals from the $\mathrm{X}, \mathrm{Y}$, and $\mathrm{Z}$ arms of the antenna is filtered through a sharp 30-100 $\mathrm{MHz}$ passive analog filter. After filtering, the signal is split into two parts: one allows building the trigger by direct comparison to a threshold level which can be remotely controlled by the user. The second signal is fed into an AD8310 logarithmic amplifier, which creates an envelope of the signal. The obtained signal-envelope contains most of the physical information of the EAS induced radio signal, while the fast ( $\sim 20 \mathrm{~ns}$ period) variations of the signal at the antenna output, which are simply due to the antenna filtering, are removed. The envelope detection performed by the logarithmic amplifier allows for a sampling of the signals at a (very comfortable) rate of 50MS/s with a 12 bits ADC without significantly altering the information quality of the EAS signal (see Figure 2). Once the on-board FPGA receives a trigger signal, it tags it through a GPS unit and sends it to the central DAQ through an optical fiber, together with a $3 \times 3.6 \mu$ s subset of digitized signals from the three channels.

In addition, the three remaining GRANDproto35 units will be equipped with electronics units initially developed for AERA by Nijmegen University and Nikhef. This low-power smart digital 

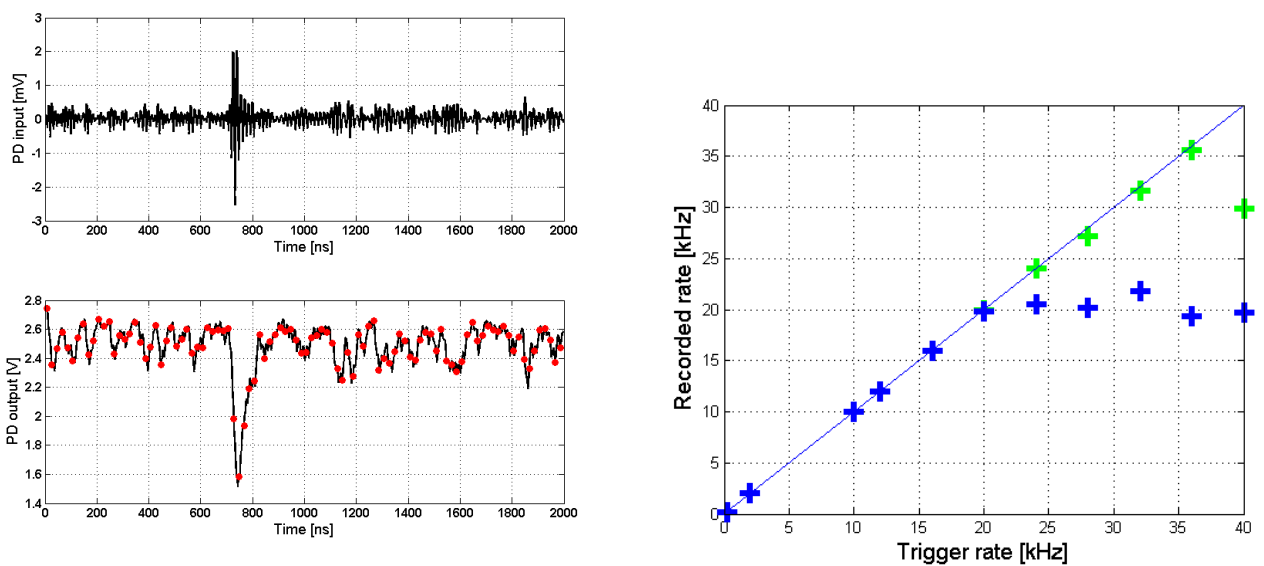

Figure 2: Left: example of an expected background event at the antenna output (top) and its simulated response by the LPNHE logarithmic amplifier (bottom). Also shown (red dots) is the sampled signal at a 50MS/s rate. Right: measured rate of recorded events with the LPNHE electronics as a function of the rate of impulses generated at the electronics input with a wave generator, for recorded pulse durations of 3.6 (blue) and $1.8 \mu \mathrm{s}$ (green).

oscilloscope [12] digitizes up to 4 input channels at $200 \mathrm{MS} / \mathrm{s}$, thus saving the complete information of each event. Using this board, $5 \mu \mathrm{s}$ of information is saved around each radio trigger, which is time-stamped using a Trimble GPS unit. The use of this electronics will allow for a direct comparison with the AERA setup in Argentina, as well as an evaluation of the power-detection method that is applied in the GRANDproto35 boards, and a cross calibration of the different types of electronics. An example radio signal obtained with the Dutch electronics during preliminary tests (see section 4) is shown in Figure 5. The figure clearly shows the low noise level recorded at the experimental site.

\subsection{Scintillator detectors}

An hybrid setup, using both radio detection and particle detection at the surface, allows for a better study of the energy and chemical composition of the cosmic rays without being contaminated by background. In addition, a well-controlled scintillator array allows for an offline verification of the performance of the radio detector.

Plastic scintillator has the advantage of combining a fairly high light output with a fast signal that has a decay time of about $2 \mathrm{~ns}$. Moreover, such a detector is easy to be operated and maintained. We have selected scintillator tiles of $70.7 \times 70.7 \times 2 \mathrm{~cm}^{3}$ as our detection unit. Each tile consists of EJ-200 plastic scintillator with four machined edges, one polished face and one sanded face. An air light-guide connects the scintillator to the Hamamatsu R7725 phototube. In order to increase the light output, the outside faces of the scintillator and the inside of the light-guide are covered with reflective Tyvek 1082D. Also the detector are mounted on a support tilted by $\sim 50^{\circ}$ from the horizontal towards North, in order to optimize the array effective area for showers coming from this direction with zenith inclination $\geq 40^{\circ}$, where the radio detection efficiency is also larger. 


\section{Principles of GRANDproto35}

The GRANDproto35 radio data acquisition will be able to record all transient signals under standard background conditions at the experimental site. An offline selection of EAS candidates from the recorded data through the search of the specific EAS signatures detailed in $[9,13]$ will provide the efficiency of this setup. Radio waves of large duration (>300 ns), curved wavefront events, or events coming from noisy directions will be rejected. A similar treatment performed by TREND allowed to isolate a nearly EAS-pure sample from an initial set of $7.3 \cdot 10^{8}$ events, at the cost of an estimated $\sim 50 \%$ EAS selection efficiency [9].

Computation of the electromagnetic wave polarization at each triggered antenna position, made possible by the recording of $\mathrm{X}, \mathrm{Y}$, and $\mathrm{Z}$ antenna voltages, provides an additional tool to discriminate EAS from background. As the wide variety of background sources makes it impossible to properly model the background, it is extremely difficult to estimate the rejection potential of radio polarization measurements, but the very specific polarization signature expected for EAS signals [14], together with preliminary measurements performed at the GRANDproto35 site (see section 4) indicate that a background discrimination treatment including polarization information should yield a background rejection better than previously achieved by TREND, combined with a significantly larger EAS detection efficiency.

The scintillator array is read-out through a parallel DAQ system. Three-fold coincidences
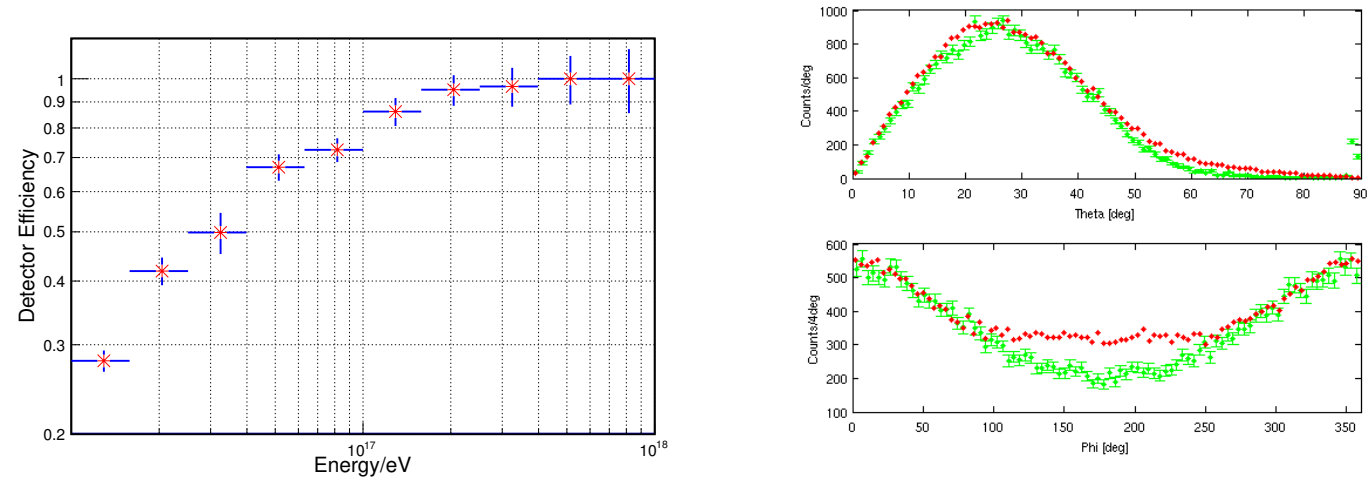

Figure 3: Left: expected efficiency of the scintillator array for cosmic ray showers with an incoming direction within $\pm 20^{\circ}$ from North, zenith angle $70^{\circ}>\theta>40^{\circ}$, an energy $\mathrm{E}>2 \cdot 10^{17} \mathrm{eV}$ and a core position inside the GRANDproto35 array. Right: zenith (top) and azimuth (bottom) distributions of events reconstructed with the 6-scintillators prototype array (see section 2.2). 3-fold coincidences are shown in red, larger multiplicities in green. The titled support of the scintillators explains the different rates measured towards North $\left(\phi=0^{\circ}\right)$ and South $\left(\phi=180^{\circ}\right)$ directions.

between scintillator triggers result in a valid scintillator event written to disk and tagged with its own GPS time information. As can be seen in Figure 3, simulations show that the scintillator array detection efficiency should be $95 \%$ for cosmic rays with an incoming direction within $\pm 20^{\circ}$ from North, zenith angle $70^{\circ}>\theta>40^{\circ}$, an energy $\mathrm{E}>2 \cdot 10^{17} \mathrm{eV}$ and a core position inside the GRANDproto35 array. The expected event rate is $\sim 1$ day $^{-1}$, while the probability for random coincidences between 3 scintillators within the time window considered is negligible. Scintillator 
events will therefore form a background-free sample of EAS events. As the radio array efficiency is also expected to reach $\sim 100 \%$ for this subset of data, an event-by-event cross check of the EAS nature of the selected radio candidates should be possible, hence allowing a quantitative and fully experimental computation of the GRANDproto35 EAS identification efficiency.

\section{Status and perspective}

Six GRANDproto35 antennas have already been deployed at the experimental site in summer 2015 to test their long-term reliability in outdoor conditions. All of these are still in perfect running condition today.

Two prototype cards of the LPNHE electronics have successfully been tested. In particular, DAQ live times larger than $95 \%$ could be measured up to $20 \mathrm{kHz}$ trigger rate (see Figure 2). Onsite tests also resulted in a $100 \%$ data recording rate obtained over a period of few hours, while long-term monitoring of the signal baseline exhibited a very stable periodic signal, consistent with Galactic plane crossing the antenna field of view (see Figure 4). Therefore, we concluded that this setup performs according to specifications in terms of sensitivity and stability. Additional 32 LPNHE cards will be produced and tested in the summer of 2017, for a deployment on site completed before the end of this year.

The Dutch AERA electronics has been deployed on site since the summer of 2016, and have

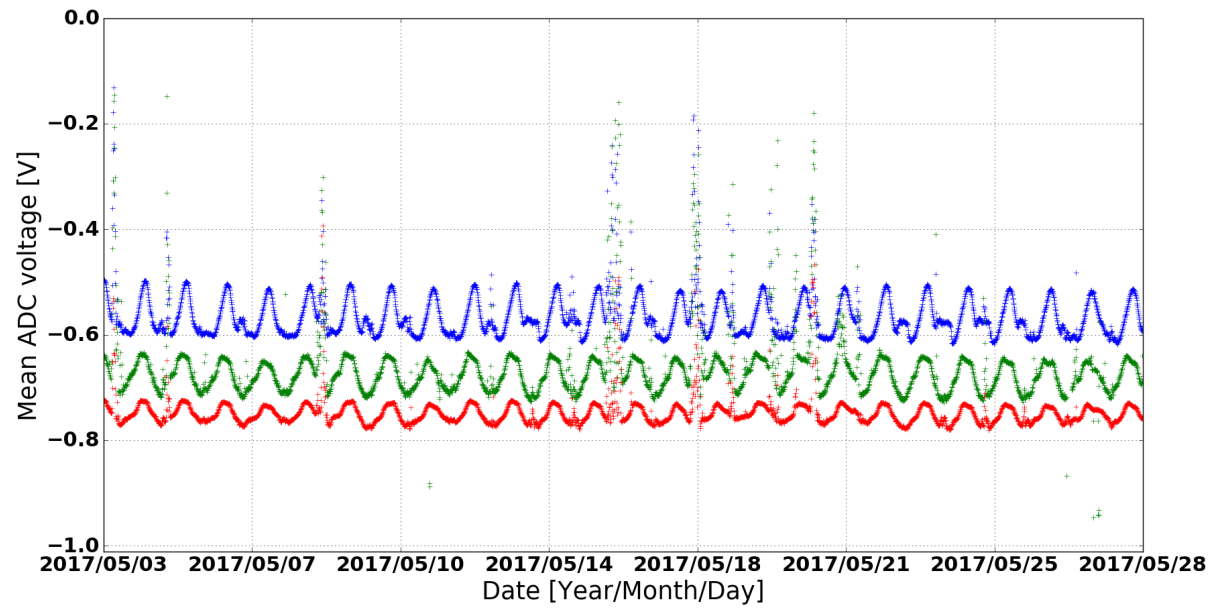

Figure 4: Monitoring measurement of the signal mean voltage for a period of 28 days at the GRANDproto35 experimental site for channels X (blue), Y (green) and Z (red) of a test detection unit. The daily-periodic fluctuations correspond to the crossing of the antenna field of view by the Galactic plane.

verified the low background conditions in Ulastai. In addition, polarization measurements were possible using this setup. An example is shown in Figure 5, showing the effectiveness of using the polarization angle, even from a single station, in order to remove background events.

Six scintillator units were deployed on site in summer 2015, and form a prototype array which 

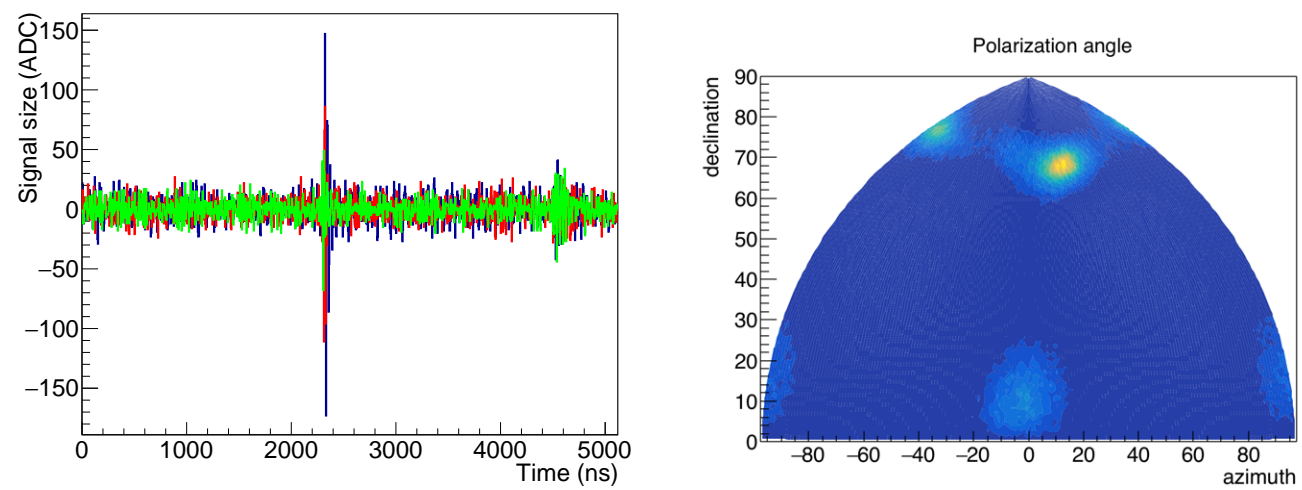

Figure 5: Left: recorded EAS induced radio candidate with the Dutch electronics. The horizontal arms are shown in red and green, the vertical arm is shown in blue. Right: polarization angle from the signals of a single station measured with the Dutch electronics.

ran for few months, allowing to test and validate DAQ and reconstruction software. In particular reconstruction of the directions of arrival of 3-fold (or more) coincidences resulted in the expected azimuthal and zenithal distributions (see Figure 3).

Array deployment will be completed in the next months, allowing the GRANDproto35 array to start taking data early 2018.

\section{Conclusion}

The GRANDproto35 array is an autonomous radio-array optimized for the self-detection of EAS. It aims at demonstrating that an efficient and background-free detection of EAS is possible with the radio technique only. An independent scintillator array deployed at the same site will provide a tool to quantify the performance of the antenna array. Developments and tests of the detector parts are now completed and deployment is beginning. The full GRANDproto35 array is expected to start taking data early 2018 .

Acknowledgements: The GRANDproto project is supported by the France China Particle Physics Laboratory, the Natural Science Foundation of China (Nos.11135010, 11105156, 11375209 and 11405180), the Chinese Ministry of Science and Technology, and the Youth Innovation Promotion Association of Chinese Academy of Sciences.

\section{References}

[1] F. Schröder, “ Radio detection of Cosmic-Ray Air Showers and High-Energy Neutrinos”, Progress in Particle and Nuclear Physics 93 (2017) 1-68

[2] A. Aab et al. [Pierre Auger Collaboration], "Energy Estimation of Cosmic Rays with the Engineering Radio Array of the Pierre Auger Observatory,” Phys. Rev. D 93 (2016) no.12, 122005 
[3] A. Aab et al. [Pierre Auger Collaboration], "Measurement of the Radiation Energy in the Radio Signal of Extensive Air Showers as a Universal Estimator of Cosmic-Ray Energy," Phys. Rev. Lett. 116 (2016) no.24, 241101

[4] S. P. Knurenko, Z. E. Petrov and I. S. Petrov, "Radio emission of air showers with extremely high energy measured by the Yakutsk radio array," doi:10.1016/j.nima.2017.04.033 arXiv:1705.01260 [astro-ph.IM].

[5] S. Buitink et al., "Method for high precision reconstruction of air shower $X_{\max }$ using two-dimensional radio intensity profiles,” Phys. Rev. D 90 (2014) no.8, 082003

[6] P.A. Bezyazeekov et al. [Tunka-Rex Collaboration], "Radio measurements of the energy and depth of maximum of cosmic-ray air showers by Tunka-Rex,” JCAP 01 (2016) 052 doi:10.1088/1475-7516/2016/01/052

[7] K. Fang et al., "The Giant Radio Array for Neutrino Detection: Present and Perspectives", these proceedings.

[8] J. Schulz [Pierre Auger Collaboration], "Status and Prospects of the Auger Engineering Radio Array," PoS ICRC 2015 (2016) 615.

[9] S. Le Coz et al., "Detection of Extensive Air Showers with the self-triggered TREND radio array", these proceedings.

[10] J. Wang et al., "How to Identify and Separate Bright Galaxy Clusters from the Low-frequency Radio Sky?,” Astrophys. J. 723 (2010) 620

[11] P. Abreu et al. [Pierre Auger Collaboration], "Antennas for the Detection of Radio Emission Pulses from Cosmic-Ray,” JINST 7 (2012) P10011

[12] J. L. Kelley [Pierre Auger Collaboration], "Data Acquisition, Triggering, and Filtering at the Auger Engineering Radio Array,” Nucl. Instrum. Meth. A 725 (2013) 133

[13] D. Ardouin et al., [TREND collaboration] "First detection of extensive air showers by the TREND self-triggering radio experiment,” Astropart. Phys. 34 (2011) 717

[14] A. Aab et al. [Pierre Auger Collaboration], "Probing the radio emission from air shower with polarization measurements", Phys. Rev. D 89 (2014) 052002 